\title{
An Efficient Synthesis of Chiral Catalyst: Application in Enantioselective Michael Addition Reactions
}

\author{
Hayriye Nevin Genc ${ }^{1 *}$ \\ ${ }^{1}$ Necmettin Erbakan University, A. K. Education Faculty, Department of Science Education, Konya, Turkey (ORCID: 0000-0003-3240-0714)
}

(İlk Geliş Tarihi 30 Temmuz 2019 ve Kabul Tarihi 27 Eylül 2019)

(DOI: 10.31590/ejosat.598343)

ATIF/REFERENCE: Genç, H. N. (2019). An Efficient Synthesis of Chiral Catalyst: Application in Enantioselective Michael Addition Reactions. Avrupa Bilim ve Teknoloji Dergisi, (17), 20-28.

\begin{abstract}
Asymmetric synthesis, also called stereoselective synthesis, chiral synthesis or enantioselective synthesis, is a form of organic synthesis which presentes one or more new elements of chirality. Use of catalysts is one of the most effective methods preferred by the researchers in recent years. It causes to the preferential formation of a stereoisomer rather than a constitutional isomer. In this study, tetraoxocalix[2] arene[2]triazine was synthesized firstly by using resorcinol and cyanuric chloride and then this starting material was derivatized with $(R)-(-)-2$-phenylglycinol to obtain tetraoxocalix[2]arene[2]triazine based chiral compound. Characterization of tetraoxocalix[2] arene[2] triazine was done by ${ }^{1} \mathrm{H}$ and ${ }^{13} \mathrm{C}$ NMR spectroscopy which is depicted in literature. Then, new organocatalyst, which possess an amino and a hydroxyl group, was designed and synthesized. The structure of the receptor characterized by FTIR, ${ }^{1} \mathrm{H}$ spectroscopy, ${ }^{13} \mathrm{C}$ NMR spectroscopy, optical rotation and elemental analysis measurement was also included. The newly prepared tetraoxocalix[2] arene[2]triazine derivative was employed as a chiral ligand in the enantioselective Michael addition of dimethylmalonate to conjugated nitroalkenes and good to excellent enantioselectivities were obtained. Various factors, (solvent, temperature, catalyst \%) were examined and the reactions were optimized. The best condition for the Michael addition reaction was determined as room temperature, toluene as solvent and $10 \mathrm{~mol} \%$ of heteroatom-bridged calixaromatic based chiral catalyst as organocatalyst loading. The catalytic efficiency of the chiral catalyst was analyzed by HPLC using chiral columns. The corresponding adducts were generally obtained in $(S)$-forms with great yields (up to 93\%) and enantioselectivities (up to $95 \%$ ee).
\end{abstract}

Keywords: Asymmetric synthesis, Organocatalysis, Michael addition, Stereoselectivity

\section{Etkili Bir Kiral Katalizör Sentezi: Enantiyoselektif Michael Reaksiyonlarında Kullanılması}

Öz

Kiral sentez, enantioselektif sentez veya stereoselektif sentez olarak da adlandırılan asimetrik sentez, stereoseçici olarak bir veya daha fazla kiral bileşiklerin oluşturulmasını sağlayan organik sentez biçimidir. Bir molekülün farklı enantiyomerleri veya diastereomerleri çoğu zaman farklı biyolojik aktiviteye sahip olduğu için asimetrik sentez özellikle ilaç endüstrisinde ve organik kimya alanında önemlidir. Kiral katalizör kullanımı ise, son yıllarda araştırmacılar tarafından tercih edilen en etkili yöntemlerden birisi olup; normal şartlarda rasemik karışım şeklinde ürün veren bir tepkimeye etki ederek, ağırlıklı olarak bir stereoizomeri elde etmeye yönelik bir stratejidir. Bu çalışmada ilk önce tetraoksakaliks[2]aren[2]triazin resorsinol ve siyanürik klorid kullanılarak sentezlendi ve sonra bu başlangıç maddesi (R)-(-)-2-fenilglisinol ile reaksiyona sokularak tetraoksakaliks[2]aren[2]triazin bazlı kiral bir bileşik elde edildi. Tetraoksakaliks[2] aren[2] triazinin karakterizasyonu, literatürde bulunan ${ }^{1} \mathrm{H}$ ve ${ }^{13} \mathrm{C}$ NMR spektroskopi değerleri ile karşılaştırılarak yapıldı. Daha sonra, hidroksil ve amino grubuna sahip yeni bir organokatalizör tasarlandı ve sentezlendi. Yeni katalizörün yapısı ${ }^{1} \mathrm{H}$ ve

${ }^{*}$ Corresponding Author: Necmettin Erbakan University, A. K. Education Faculty, Department of Science Education, Konya, Turkey, ORCID: 00000003-3240-0714, hngenc@erbakan.edu.tr 
${ }^{13} \mathrm{C}$ NMR spektroskopisi, elemental analiz ve çevirme açısı teknikleriyle aydınlatıldı. Sentezlenen kiral bileşik dimetilmalonatın farklı nitroalkenlerle olan enantiyoselektif Michael katılma reaksiyonlarında katalizör olarak kullanıldı ve çok iyi enantiyoselektivite elde edildi. Reaksiyonu etkileyen farklı faktörler (çözücü, sıcaklık, katalizör yüzdesi) incelenerek reaksiyon şartları optimize edildi. En iyi reaksiyon şartları oda sıcaklığı, çözücü olarak toluen ve \%10 mol katalizör olarak bulundu. Kiral katalizörün katalitik etkisi HPLC'de kiral kolonlar kullanılarak belirlendi. Reaksiyon sonucunda oluşan ürünler yüksek verim (\%93) ve yüksek enantiyomerik fazlalık $(\% 95)$ ile $(S)$ formunda elde edildi.

Anahtar Kelimeler: Asimetrik sentez, Organokatalizör, Michael katılma, Stereoselektivite

\section{Introduction}

Chirality has an important place in the life of animals and plants, agriculture, medicine and chemical industry. Chiral compound synthesis methods are one of the most important follow-up areas of synthetic chemistry for the production of agricultural chemicals and functional materials in pharmaceuticals (Comelles et al., 2005; Harutyunyan et al., 2008; Sulzer-Mossé S and Alexakis, 2007). Generally, chirality introduces authenticity and a remarkable selectivity in the activities of the drug. In the case of an enantiomer active in the drug, the other enantiomer may be inactive, less active or toxic (Enders et al., 2006; Almasi et al., 2007).

Therefore it is a great importance to obtain products in enantiomeric purity. Generally, studies are about synthesizing single enantiomers of chiral molecules (Li et al., 2010; Genc and Sirit, 2018). There are three general ways that can be used for this purpose. The first of these; separation of racemic mixtures by different methods; the synthesis of single enantiomers using chiral auxiliaries and the third route; is the use of chiral catalysts during the synthesis of chiral molecules. In all three methods, the goal is to realize asymmetric synthesis of molecules as efficiently as possible in an enantiospecific, high yield and as cheap as possible (Tsakos et al., 2012; Tsogoeva, 2007; Ma et al., 2010).

Organocatalysts are organic compounds that accelerate chemical reactions. Due to their function in organic synthesis, the enantioselective Michael addition reactions catalyzed by organocatalysts have progressed considerably and are gaining in importance in recent times (Yalalov et al., 2006; Lu et al., 2012; Hayashi et al., 2005). Asymmetric conjugate addition reactions in which active methylene is used as an acceptor of the donor and $\alpha, \beta$-unsaturated compounds are among the most important of the carbon-carbon and carbon-heteroatom bond forming reactions (Naziroglu et al., 2012; Mase et al., 2006; Mase et al., 2004; Liu et al., 2013).

Today, it is obvious that the advantages of asymmetric organocatalysis creates a challenging research area (Tsandi et al., 2009; Nugent et al., 2011; Palomo et al., 2006; Pansare and Pandya, 2006). Hence, the importance of using chiral catalysts has been growing. The increased possibility of the synthesis of enantiopure intermediates for the production of pharmaceuticals keeps the eyes on organocatalysis. There are also so many advantages such as shorter reaction duration, lower impurity and inexpensive processes (Yoshida et al., 2011; Dong et al., 2012; Naziroglu and Sirit, 2016).

Tetraoxacalix[2] arene[2]triazine derivatives bearing diffirent functionalities have also reported as asymmetric organocatalysts recently (Genc et al., 2019a; Genc et al., 2019b; Genc, 2019). In the first part of study new tetraoxa calix[2]arene[2]triazine derivative 5 was designed and synthesized. After having synthesized the chiral catalyst, trans- $\beta$-nitrostyrene (6) was initially reacted with dimethyl malonate (7) using 5 to explore the catalytic properties of the product.

In the second part of the study, asymmetric Michael Addition reaction were optimized by testing chiral catalyst and solvent effect, reaction temperature as well as catalyst loading. These optimized parameters were used for the reaction of asymmetric Michael addition of 7 to various aromatic nitroalkenes affording the Michael adducts 8a-81 in 85-93\% yields and with excellent enantioselectivities (78-95\%). The sythesis and application of calix[2]arene[2]triazine $(R)-(+)-2-$ Phenylglycinol derivative is first reported in asymmetric Michael reaction.

\section{Materials and Methods}

\subsection{Synthesis of compound 5}

Tetraoxacalix[2] arene[2]triazine 4 was sythesized following a method published by Wang \& Yang (2004) and Bozkurt \& Türkmen (2016). A mixture of (R)-(-)-2-phenylglycinol $(1.1 \mathrm{mmol})$ and N,N-Diisopropylethylamine (2.2 mmol) in tetrahydrofuran $(20 \mathrm{~mL})$ was added to a solution of compound $4(0.5 \mathrm{mmol})$ in THF $(20 \mathrm{~mL})$. The mixture was heated at reflux for $18-20 \mathrm{~h}$, after which time the THF was evaporated until solid remains. The remaining mixture was purified by column chromatography technique using hexane/ethyl acetate $(1: 8, \mathrm{v} / \mathrm{v})$. Product $5{ }^{1} \mathrm{H} \mathrm{NMR}\left(400 \mathrm{MHz}, \mathrm{CDCl}_{3}\right)$ and ${ }^{13} \mathrm{C} \mathrm{NMR}\left(100 \mathrm{MHz}, \mathrm{CDCl}_{3}\right)$ spectra were shown in figure 1.

Compound 5 Yellow crystalline solid; 71\% yield; $[\alpha]_{\mathrm{D}}^{20}+63.90\left(c 1, \mathrm{CHCl}_{3}\right) ; \mathrm{mp} 138-140{ }^{\circ} \mathrm{C}$; IR $\left(\mathrm{cm}^{-1}\right): 3392,3291,2951,1577$, 1375; ${ }^{1} \mathrm{H}$ NMR $\left(400 \mathrm{MHz}, \mathrm{CDCl}_{3}\right): \delta=7.38-7.34(\mathrm{~m}, 2 \mathrm{H}), 7.32-7.25(\mathrm{~m}, 4 \mathrm{H}), 7.12-7.05(\mathrm{~m}, 2 \mathrm{H}), 6.79-6.66(\mathrm{~m}, 6 \mathrm{H}), 6.62-6.51(\mathrm{~m}$, $4 \mathrm{H}), 5.40-5.33(\mathrm{~m}, 2 \mathrm{H}), 4.35-4.29(\mathrm{~m}, 2 \mathrm{H}), 4.25-4.16(\mathrm{~m}, 2 \mathrm{H}), \mathrm{NH}$ and $\mathrm{OH}$-signals not determined; ${ }^{13} \mathrm{C} \mathrm{NMR}(100 \mathrm{MHz}, \mathrm{CDCl} 3): \delta=$ 171.43, 169.13, 165.73, 152.13, 139.40, 138.60, 128.31, 126.79, 119.28, 116.27, 71.88, 62.00, 60.50, 55.90, 31.58, 27.90; Anal. Calcd. for $\mathrm{C}_{34} \mathrm{H}_{28} \mathrm{~N}_{8} \mathrm{O}_{6}$ (644.63): C, 63.34; H, 4.37; N, 17.38\%. Found: C, 63.18; H, 4.45; N, 17.22\%. 

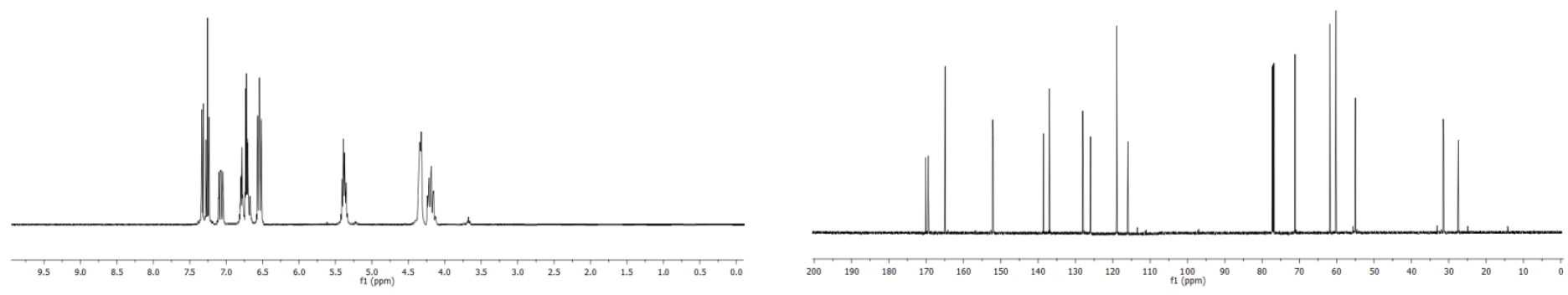

Figure 1. Product $5{ }^{1} \mathrm{H}$ NMR (400 MHz, $\left.\mathrm{CDCl}_{3}\right)$ and ${ }^{13} \mathrm{C} \mathrm{NMR}\left(100 \mathrm{MHz}, \mathrm{CDCl}_{3}\right)$ spectra

\subsection{Typical procedure for Michael addition reactions}

Catalyst 5 (0.1 equiv.) was added to a stirred solution of trans- $\beta$-nitrostyrene (1.0 equiv.) and dimethyl malonate (3.0 equiv.) in toluene $(0.2 \mathrm{~mL})$ at r.t. After mixing for 36-48 hours, the solid residue was purified (elution gradient: EtOAc: Hexane $=1: 10)$ to afford desired Michael adduct 8a-81. Products HPLC conditions are shown in the Table 1 and FTIR, ${ }^{1} \mathrm{H}$ NMR and ${ }^{13} \mathrm{C}$ NMR values are shown in Table 2.

Table 1. Product $8 \mathbf{8}-8 \mathbf{l}$ HPLC conditions

\begin{tabular}{|l|l|l|l|l|l|}
\hline Product & Column & $\begin{array}{l}\text { Hexane/ } \\
\text { 2-propanol }\end{array}$ & $\begin{array}{l}\text { Flow rate } \\
(\mathbf{m L} / \mathbf{m i n})\end{array}$ & $\begin{array}{l}\mathbf{t}_{\boldsymbol{R}}(\mathbf{m i n o r}) \\
(\mathbf{m i n})\end{array}$ & $\begin{array}{l}\mathbf{t}_{\boldsymbol{R}} \text { (major) } \\
(\mathbf{m i n})\end{array}$ \\
\hline $\mathbf{8 a}$ & AD-H & $90: 10$ & 1.0 & 18.2 & 29.5 \\
\hline $\mathbf{8 b}$ & AD-H & $90: 10$ & 1.0 & 19.2 & 12.5 \\
\hline $\mathbf{8 c}$ & OD-H & $90: 10$ & 1.0 & 20.8 & 23.1 \\
\hline $\mathbf{8 d}$ & AD-H & $75: 25$ & 1.5 & 7.5 & 10.9 \\
\hline $\mathbf{8 e}$ & AD-H & $70: 30$ & 1.0 & 8.1 & 10.3 \\
\hline $\mathbf{8 f}$ & AD-H & $70: 30$ & 1.0 & 7.9 & 14.0 \\
\hline $\mathbf{8 g}$ & OD-H & $70: 30$ & 0.5 & 15.3 & 19.5 \\
\hline $\mathbf{8 h}$ & OD-H & $70: 30$ & 0.5 & 24.1 & 28.2 \\
\hline $\mathbf{8 i}$ & AD-H & $70: 30$ & 1.0 & 11.6 & 19.3 \\
\hline $\mathbf{8 j}$ & OD-H & $70: 30$ & 0.9 & 13.9 & 20.6 \\
\hline $\mathbf{8 k}$ & AD-H & $75: 25$ & 1.0 & 9.9 & 14.0 \\
\hline $\mathbf{8 1}$ & OD-H & $85: 15$ & 0.5 & 35.2 & 39.8 \\
\hline
\end{tabular}


Table 2. Product 8a-8l FTIR, ${ }^{l} \mathrm{H} N M R\left(400 \mathrm{MHz}, \mathrm{CDCl}_{3}\right)$ and ${ }^{13} \mathrm{C} N M R\left(100 \mathrm{MHz}, \mathrm{CDCl}_{3}\right)$ spectroscopy values.

\begin{tabular}{|c|c|c|}
\hline \multirow{3}{*}{ 8a } & $\operatorname{IR}\left(\mathrm{cm}^{-1}\right)$ & $2942,1713,1542,1485,1435,1365,1241,1055,982,744,675$ \\
\hline & ${ }^{1} \mathrm{H}$ NMR & $\begin{array}{l}7.27-7.16(\mathrm{~m}, 5 \mathrm{H}), 4.91-4.83(\mathrm{~m}, 2 \mathrm{H}), 4.21-4.18(\mathrm{~m}, 1 \mathrm{H}), 3.82(\mathrm{~d}, J=8.9 \mathrm{~Hz}, 1 \mathrm{H}), 3.72 \\
(\mathrm{~s}, 3 \mathrm{H}), 3.48(\mathrm{~s}, 3 \mathrm{H})\end{array}$ \\
\hline & ${ }^{13} \mathrm{C} \mathrm{NMR}$ & $168.7,168.2,137.5,129.9,129.6,128.9,78.6,55.8,54.3,53.8,44.0$ \\
\hline \multirow{3}{*}{$8 \mathbf{b}$} & $\operatorname{IR}\left(\mathrm{cm}^{-1}\right)$ & $2943,1724,1541,1421,1144,1009,732$ \\
\hline & ${ }^{1} \mathrm{H}$ NMR & $\begin{array}{l}7.51-7.49(\mathrm{~m}, 1 \mathrm{H}), 7.21-7.11(\mathrm{~m}, 2 \mathrm{H}), 7.10-7.02(\mathrm{~m}, 1 \mathrm{H}), 5.04-4.99(\mathrm{~m}, 1 \mathrm{H}), 4.90-4.85 \\
(\mathrm{~m}, 1 \mathrm{H}), 4.70-4.66(\mathrm{~m}, 1 \mathrm{H}), 4.00(\mathrm{~d}, J=8.1 \mathrm{~Hz}, 1 \mathrm{H}), 3.65(\mathrm{~s}, 3 \mathrm{H}), 3.58(\mathrm{~s}, 3 \mathrm{H})\end{array}$ \\
\hline & ${ }^{13} \mathrm{C}$ NMR & $168.6,168.0,136.1,134.9,131.8,128.8,125.6,76.5,54.1,53.2,42.1$ \\
\hline \multirow{3}{*}{$8 \mathbf{c}$} & $\operatorname{IR}\left(\mathrm{cm}^{-1}\right)$ & $672,770,810,996,1069,1260,1371,1478,1549,1719,2720,2819,2961$ \\
\hline & ${ }^{1} \mathrm{H} \mathrm{NMR}$ & $\begin{array}{l}7.53-7.49(\mathrm{~m}, 2 \mathrm{H}), 7.30-7.25(\mathrm{~m}, 2 \mathrm{H}), 5.01-4.92(\mathrm{~m}, 2 \mathrm{H}), 4.34-4.27(\mathrm{~m}, 1 \mathrm{H}), 3.91(\mathrm{~d}, J= \\
8.9 \mathrm{~Hz}, 1 \mathrm{H}), 3.81(\mathrm{~s}, 3 \mathrm{H}), 3.67(\mathrm{~s}, 3 \mathrm{H})\end{array}$ \\
\hline & ${ }^{13} \mathrm{C} \mathrm{NMR}$ & $166.8,166.1,137.5,131.6,130.7,128.5,125.8,122.0,76.1,53.9,52.4,52.0,41.8$ \\
\hline \multirow{3}{*}{ 8d } & $\operatorname{IR}\left(\mathrm{cm}^{-1}\right)$ & $2965,2931,1763,1551,1493,1441,1352,1165,1019,836$ \\
\hline & ${ }^{1} \mathrm{H}$ NMR & $\begin{array}{l}7.40-7.37(\mathrm{~m}, 2 \mathrm{H}), 7.06-7.04(\mathrm{~m}, 2 \mathrm{H}), 4.87-4.75(\mathrm{~m}, 2 \mathrm{H}), 4.18-4.11(\mathrm{~m}, 1 \mathrm{H}), 3.78(\mathrm{~d}, J= \\
8.9 \mathrm{~Hz}, 1 \mathrm{H}), 3.71(\mathrm{~s}, 3 \mathrm{H}), 3.52(\mathrm{~s}, 3 \mathrm{H})\end{array}$ \\
\hline & ${ }^{13} \mathrm{C} \mathrm{NMR}$ & $167.0,166.4,134.3,131.6,128.9,121.9,76.5,53.7,52.6,52.1,41.8$ \\
\hline \multirow{3}{*}{$8 \mathrm{e}$} & $\operatorname{IR}\left(\mathrm{cm}^{-1}\right)$ & $2941,2842,1721,1542,1482,1422,1246$ \\
\hline & ${ }^{1} \mathrm{H} \mathrm{NMR}$ & $\begin{array}{l}7.37-7.26(\mathrm{~m}, 2 \mathrm{H}), 7.20-7.08(\mathrm{~m}, 2 \mathrm{H}), 4.99(\mathrm{~d}, J=6.9 \mathrm{~Hz}, 2 \mathrm{H}), 4.54-4.48(\mathrm{~m}, 1 \mathrm{H}), 4.06 \\
(\mathrm{~d}, J=9.6 \mathrm{~Hz}, 1 \mathrm{H}), 3.79(\mathrm{~s}, 3 \mathrm{H}), 3.62(\mathrm{~s}, 3 \mathrm{H})\end{array}$ \\
\hline & ${ }^{13} \mathrm{C} \mathrm{NMR}$ & $167.2165 .8,160.6,158.1,130.1,123.2,121.9,115.0,74.5,74.1,51.9,51.2,50.1,36.6$ \\
\hline \multirow{3}{*}{$8 f$} & $\operatorname{IR}\left(\mathrm{cm}^{-1}\right)$ & $3023,2965,2921,1748,1720,1568,1486,1395,915,748,663$ \\
\hline & ${ }^{1} \mathrm{H}$ NMR & $\begin{array}{l}7.20-7.15(\mathrm{~m}, 2 \mathrm{H}), 6.98-6.94(\mathrm{~m}, 2 \mathrm{H}), 4.88-4.74(\mathrm{~m}, 2 \mathrm{H}), 4.21-4.16(\mathrm{~m}, 1 \mathrm{H}), 3.75(\mathrm{~d}, J= \\
9.1 \mathrm{~Hz}, 1 \mathrm{H}), 3.70(\mathrm{~s}, 3 \mathrm{H}), 3.51(\mathrm{~s}, 3 \mathrm{H})\end{array}$ \\
\hline & ${ }^{13} \mathrm{C} \mathrm{NMR}$ & $168.6,164.3,162.1,132.6,132.1,130.0,114.9,114.1,76.6,53.8,52.2,51.1,41.2$ \\
\hline \multirow{3}{*}{$8 \mathrm{~g}$} & $\mathrm{IR}\left(\mathrm{cm}^{-1}\right)$ & $2912,2241,1785,1541,1488,1376,1239,1145,1081,900,721,633$ \\
\hline & ${ }^{1} \mathrm{H} \mathrm{NMR}$ & $\begin{array}{l}7.24-7.18(\mathrm{~m}, 1 \mathrm{H}), 7.11(\mathrm{dd}, J=1.7 \mathrm{~Hz}, 7.9 \mathrm{~Hz}, 1 \mathrm{H}), 6.85-6.81(\mathrm{~m}, 2 \mathrm{H}), 5.02-4.95(\mathrm{~m}, \\
1 \mathrm{H}), 4.86-4.81(\mathrm{~m}, 1 \mathrm{H}) 4.38-4.32(\mathrm{~m}, 1 \mathrm{H}), 4.14(\mathrm{~d}, J=9.8 \mathrm{~Hz}, 1 \mathrm{H}), 3.78(\mathrm{~s}, 3 \mathrm{H}), 3.69(\mathrm{~s}, \\
3 \mathrm{H}), 3.47(\mathrm{~s}, 3 \mathrm{H})\end{array}$ \\
\hline & ${ }^{13} \mathrm{C} \mathrm{NMR}$ & $169.1,168.4,158.1,131.2,129.7,124.4,121.6,112.0,77.3,56.2,53.6,53.1,41.1$ \\
\hline \multirow{3}{*}{$8 \mathbf{h}$} & $\operatorname{IR}\left(\mathrm{cm}^{-1}\right)$ & $2942,2861,1752,1622,1571,1485,1273,106$ \\
\hline & ${ }^{1} \mathrm{H}$ NMR & $\begin{array}{l}7.22-7.18(\mathrm{~m}, 1 \mathrm{H}), 6.79-6.76(\mathrm{~m}, 3 \mathrm{H}), 4.90-4.81(\mathrm{~m}, 2 \mathrm{H}), 4.21-4.14(\mathrm{~m}, 1 \mathrm{H}), 3.85(\mathrm{~d}, J= \\
9.0 \mathrm{~Hz}, 1 \mathrm{H}), 3.74(\mathrm{~s}, 3 \mathrm{H}), 3.71(\mathrm{~s}, 3 \mathrm{H}), 3.53(\mathrm{~s}, 3 \mathrm{H})\end{array}$ \\
\hline & ${ }^{13} \mathrm{C} \mathrm{NMR}$ & $168.1,158.3,136.1,128.9,118.2,111.6,76.0,54.0,53.2,52.1,51.3,41.3$ \\
\hline \multirow{3}{*}{ 8i } & $\operatorname{IR}\left(\mathrm{cm}^{-1}\right)$ & $3002,2943,2830,1721,1543,1422,1242,1023$ \\
\hline & ${ }^{1} \mathrm{H}$ NMR & $\begin{array}{l}7.10-7.05(\mathrm{~m}, 2 \mathrm{H}), 6.78-6.75(\mathrm{~m}, 2 \mathrm{H}), 4.84-4.73(\mathrm{~m}, 2 \mathrm{H}), 4.15-4.09(\mathrm{~m}, 1 \mathrm{H}), 3.76(\mathrm{~d}, J= \\
9.1 \mathrm{~Hz}, 1 \mathrm{H}), 3.70(\mathrm{~s}, 3 \mathrm{H}), 3.67(\mathrm{~s}, 3 \mathrm{H}), 3.49(\mathrm{~s}, 3 \mathrm{H})\end{array}$ \\
\hline & ${ }^{13} \mathrm{C} \mathrm{NMR}$ & $169.7,169.0,161.2,128.8,115.6,77.5,54.0,53.8,52.6,42.1$ \\
\hline \multirow{3}{*}{ 8j } & $\operatorname{IR}\left(\mathrm{cm}^{-1}\right)$ & $3005,2943,1720,1542,1423,1345$ \\
\hline & ${ }^{1} \mathrm{H}$ NMR & $\begin{array}{l}7.98(\mathrm{dd}, J=1.3 \mathrm{~Hz}, 8.3 \mathrm{~Hz}, 1 \mathrm{H}), 7.64-7.60(\mathrm{~m}, 1 \mathrm{H}), 7.55-7.51(\mathrm{~m}, 1 \mathrm{H}), 7.46-7.44(\mathrm{~m}, \\
1 \mathrm{H}), 5.20(\mathrm{dd}, J=8.3 \mathrm{~Hz}, 14.0 \mathrm{~Hz}, 1 \mathrm{H}), 5.08(\mathrm{dd}, J=4.4 \mathrm{~Hz}, 14.1 \mathrm{~Hz}, 1 \mathrm{H}), 4.80(\mathrm{td}, J= \\
4.6 \mathrm{~Hz}, 8.2 \mathrm{~Hz}, 1 \mathrm{H}), 4.28(\mathrm{~d}, J=8.0 \mathrm{~Hz}, 1 \mathrm{H}), 3.79(\mathrm{~s}, 3 \mathrm{H}), 3.68(\mathrm{~s}, 3 \mathrm{H})\end{array}$ \\
\hline & ${ }^{13} \mathrm{C}$ NMR & $169.7,151.9,135.3,133.1,131.3,130.8,127.5,77.9,55.4,55.1,39.5$ \\
\hline \multirow{3}{*}{$\mathbf{8 k}$} & $\operatorname{IR}\left(\mathrm{cm}^{-1}\right)$ & $3031,2972,2265,1799,1565,1392,1260,1103,915,743,665$ \\
\hline & ${ }^{1} \mathrm{H}$ NMR & $\begin{array}{l}7.19-7.13(\mathrm{~m}, 4 \mathrm{H}), 4.97-4.88(\mathrm{~m}, 2 \mathrm{H}), 4.27(\mathrm{dt}, J=5.4 \mathrm{~Hz}, 9.1 \mathrm{~Hz}, 1 \mathrm{H}), 3.89(\mathrm{~d}, J=9.2 \\
\mathrm{Hz}, 1 \mathrm{H}), 3.79(\mathrm{~s}, 3 \mathrm{H}), 3.63(\mathrm{~s}, 3 \mathrm{H}), 2.36(\mathrm{~s}, 3 \mathrm{H})\end{array}$ \\
\hline & ${ }^{13} \mathrm{C} \mathrm{NMR}$ & $168.8,137.1,132.0,128.6,126.5,76.2,53.6,52.0,51.6,41.4,20.0$ \\
\hline \multirow{3}{*}{81} & $\mathrm{IR}\left(\mathrm{cm}^{-1}\right)$ & $3170,2943,2269,1810,1582,1480,1395,922,665$ \\
\hline & ${ }^{1} \mathrm{H}$ NMR & $\begin{array}{l}7.36-7.33(\mathrm{~m}, 2 \mathrm{H}), 7.23-7.20(\mathrm{~m}, 2 \mathrm{H}), 4.97-4.86(\mathrm{~m}, 2 \mathrm{H}), 4.29-4.24(\mathrm{~m}, 1 \mathrm{H}), 3.86(\mathrm{~d}, J= \\
9.1 \mathrm{~Hz}, 1 \mathrm{H}), 3.78(\mathrm{~s}, 3 \mathrm{H}), 3.64(\mathrm{~s}, 3 \mathrm{H})\end{array}$ \\
\hline & ${ }^{13} \mathrm{C} \mathrm{NMR}$ & $169.4,168.0,135.4,135.1,130.1,129.2,78.0,55.2,54.0,53.2,43.5$ \\
\hline
\end{tabular}

\section{Results and Discussion}

Tetraoxacalix[2]arene[2]triazine $\mathbf{4}$ was synthesized by the reaction of resorcinol $\mathbf{1}$ with cyanuric chloride $\mathbf{2}$ in THF in two steps. Characterization of compound 4 was done by ${ }^{1} \mathrm{H}$ spectroscopy and ${ }^{13} \mathrm{C}$ NMR spectroscopy which is depicted in literature. After, compound 5 was sythesized by reacting 4 with 2.2 equiv of $(R)-(-)-2$-phenylglycinol respectively in $71 \%$ yield according to the 
synthetic procedure shown in figure 2 . The obtained products structures were also identified by ${ }^{1} \mathrm{H} N M R,{ }^{13} \mathrm{C}$ NMR and FTIR spectroscopic techniques.<smiles>Oc1cccc(O)c1</smiles>

1<smiles>Clc1nc(Cl)nc(Cl)n1</smiles>

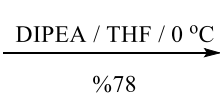<smiles>Clc1nc(Cl)nc(Oc2cccc(Oc3nc(Cl)nc(Cl)n3)c2)n1</smiles>
2<smiles>OC[C@@H](Nc1nc2nc(n1)Oc1cccc(c1)Oc1nc(N[C@@H](CO)c3ccccc3)nc(n1)Oc1cccc(c1)O2)c1ccccc1</smiles>

5

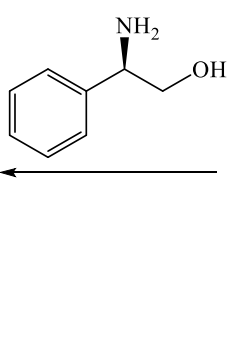<smiles>Clc1nc2nc(n1)Oc1cccc(c1)Oc1nc(Cl)nc(n1)Oc1cccc(c1)O2</smiles>

Figure 2. Procedure for the synthesis of chiral catalyst 5

Investigation was started with dimethyl malonate and nitroalkenes as a role model reaction. At the first part, we tested the effect of the solvent for maximum enantioselectivities and yields using 5 (10 mol\%). Solvents screening experiments were conducted with 12 different solvents. Among the solvents, toluene, $i$-PrOH and $\mathrm{CCl}_{4}$ gave appropriate results as compared to other solvents. Toluene was determined to be the best solvent among solvents for the reaction of dimethyl malonate and trans- $\beta$-nitrostyrene (Table 3 , entry 8).

As the second parameter, the effect of temperature was screened. Therefore the reaction was repeated at room temperature, $0{ }^{\circ} \mathrm{C}$ and $40{ }^{\circ} \mathrm{C}$. The best values were observed at r.t. compared to at $0{ }^{\circ} \mathrm{C}$ and $40{ }^{\circ} \mathrm{C}$. Reducing the reaction temperature from $25{ }^{\circ} \mathrm{C}$ to 0 ${ }^{\circ} \mathrm{C}$, decreases enantioselectivity and yield as expected (Table 3, entry 13). When the temperature raised to $40{ }^{\circ} \mathrm{C}$, in chemical yields and enantioselectivity drastic decrease was observed (Table 3, entry 14 ).

Finally, the effect of catalyst amount was studied on the reaction of dimethylmalonate to trans- $\beta$-nitrostyrene, in order to determine the most optimal reaction conditions and the results indicated a bit loss in yields and stereoselectivities when catalyst loading percent increased to $15 \mathrm{~mol} \%$. It was observed that there was a decrease in terms of enantioselectivity from $95 \%$ to $92 \%$ when the amount of organocatalyst was increased $15 \mathrm{~mol} \%$. (Table 3, entry 15 ). 
Table 3. Enantioselective Michael reaction of dimethylmalonate with trans- $\beta$-nitrostyrene catalysed by 5<smiles>O=[N+]([O-])C=Cc1ccccc1</smiles>

6<smiles>COC(=O)CC(=O)OC</smiles>

7<smiles>COC(=O)C(C(=O)OC)[C@H](C[N+](=O)[O-])c1ccccc1</smiles>

8

\begin{tabular}{|c|c|c|c|c|c|}
\hline Entr & Catalyst & Solvent & Time (d) & Yield (\%) & $\boldsymbol{e e}(\boldsymbol{\%})$ \\
\hline 1 & $\mathbf{5}$ & $i-\mathrm{PrOH}$ & 3 & 90 & $92(S)$ \\
\hline 2 & $\mathbf{5}$ & $\mathrm{CCl}_{4}$ & 3 & 88 & $90(S)$ \\
\hline 3 & $\mathbf{5}$ & $\mathrm{CHCl}_{3}$ & 4 & 82 & $83(S)$ \\
\hline 4 & $\mathbf{5}$ & $\mathrm{DMF}$ & 4 & 79 & $81(S)$ \\
\hline 5 & $\mathbf{5}$ & $\mathrm{Hexane}$ & 4 & 74 & $88(S)$ \\
\hline 6 & $\mathbf{5}$ & $\mathrm{CH}_{2} \mathrm{Cl}_{2}$ & 4 & 75 & $80(S)$ \\
\hline 7 & $\mathbf{5}$ & $\mathrm{THF}$ & 3 & 69 & $87(S)$ \\
\hline 8 & $\mathbf{5}$ & $\mathrm{Toluene}$ & 3 & 93 & $95(S)$ \\
\hline 9 & $\mathbf{5}$ & $\mathrm{EtOAc}$ & 3 & 80 & $81(S)$ \\
\hline 10 & $\mathbf{5}$ & $\mathrm{CH}_{3} \mathrm{CN}$ & 3 & 81 & $80(S)$ \\
\hline 11 & $\mathbf{5}$ & MeOH & 3 & 90 & $79(S)$ \\
\hline 12 & $\mathbf{5}$ & Et ${ }_{2} \mathrm{O}$ & 4 & 85 & $78(S)$ \\
\hline $13^{\mathrm{a}}$ & $\mathbf{5}$ & Toluene & 8 & 88 & $91(S)$ \\
\hline $14^{\mathrm{b}}$ & $\mathbf{5}$ & Toluene & 2 & 87 & $90(S)$ \\
\hline $15^{\mathrm{c}}$ & $\mathbf{5}$ & Toluene & 4 & 90 & $92(S)$ \\
\hline
\end{tabular}

${ }^{\text {a }}$ Reaction performed at $0{ }^{\circ} \mathrm{C}$.

${ }^{\mathrm{b}}$ Reaction performed at $40{ }^{\circ} \mathrm{C}$

c $15 \mathrm{~mol} \%$ catalyst was used.

As a result of all test reactions, the best conditions was determined as r.t., toluene as solvent and $10 \mathrm{~mol} \%$ of heteroatom-bridged calixaromatic based chiral catalyst $\mathbf{5}$ as organocatalyst loading. Having established the reaction conditions, using nitroolefin compounds by possessing electron-withdrawing and electron-donating groups various products were synthesized, affording the desired Michael adducts 8a-8I in 85-93\% yields and with excellent enantioselectivities (78-95\%) as demonstrated in Table 4. 
Table 4. Enantioselective Michael reaction of dimethylmalonate with various nitrostyrenes with chiral catalyst 5

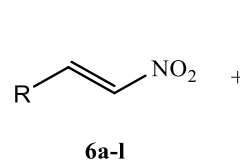<smiles>COC(=O)CC(=O)OC</smiles><smiles>C1CCCC1</smiles><smiles>[R]C(C[N+](=O)[O-])C(C(=O)OC)C(=O)OC</smiles>

\begin{tabular}{|c|c|c|c|c|}
\hline Entry & Product & Time (d) & Yield (\%) & $e e(\%)$ \\
\hline 1 & & 3 & 93 & 95 \\
\hline 2 & & 3 & 85 & 78 \\
\hline 3 & & 4 & 88 & 87 \\
\hline 4 & & 4 & 90 & 93 \\
\hline 5 & & 3 & 88 & 80 \\
\hline 6 & & 3 & 90 & 91 \\
\hline 7 & & 3 & 85 & 87 \\
\hline 8 & & 4 & 88 & 87 \\
\hline 9 & & 4 & 90 & 89 \\
\hline 10 & & 4 & 88 & 87 \\
\hline 11 & & 4 & 90 & 89 \\
\hline 12 & & 4 & 89 & 88 \\
\hline
\end{tabular}

\section{Conclusion}

In conclusion, a new type of tetraoxacalix[2]arene[2]triazine based chiral catalyst $\mathbf{5}$ was synthesized for the Michael reaction of dimethylmalonate to aromatic nitroalkenes. The optimized condition was found as $10 \mathrm{~mol} \%$ chiral catalyst 5 in toluene at room temperature. From the results, the best yield (up to $93 \%$ ) and enantiomeric excess (up to $95 \%$ ) was obtained with trans- $\beta$-nitrostyrene substrate. 
European Journal of Science and Technology

\section{References}

Almasi, D., Alonso, D. A., Najera, C. (2007). Organocatalytic asymmetric conjugate additions. Tetrahedron Asymmetry, 18, $299-365$.

Bozkurt, S., Türkmen, M. B. (2016). New chiral oxo-bridged calix[2]arene[2]triazine for the enantiomeric recognition of $\alpha$-racemic carboxylic acids. Tetrahedron Asymmetry, 27, 443-447.

Comelles, J., Moreno-Mañas, M., Vallribera, A. (2005). Michael additions catalyzed by transition metals and lanthanides species. A review. Part 1. Transition metals. ARKIVOC, 9, 207-238.

Dong, Z., Qiu, G., Zhou, H-B., Dong, C. (2012). Chiral squaramide as multiple H-bond donor organocatalysts for the asymmetric Michael addition of 1,3-dicarbonyl compounds to nitroolefins. Tetrahedron Asymmetry, 23, 1550-1556.

Enders, D., Saint-Dizier, A., Lannou, M-I., Lenzen, A. (2006). The phospha-Michael addition in organic synthesis. European Journal of Organic Chemistry, 29-49.

Genc, H. N, Sirit, A, (2018). Novel and highly efficient bifunctional calixarene thiourea derivatives as organocatalysts for enantioselective Michael reaction of nitroolefins with diketones. Journal of Inclusion Phenomena and Macrocyclic Chemistry, 90, 39-49.

Genc, H. N. (2019). Enantioselective Michael reaction of anthrone catalyzed by chiral tetraoxacalix[2]arene[2]triazine derivatives. RSC Advances, 9, 21063-21069.

Genc, H. N., Ozgun, U., Sirit, A. (2019). Chiral tetraoxacalix[2]arene[2]triazine-based organocatalysts for Enantioselective Aldol reactions. Tetrahedron Letters, 60, 1763-1768.

Genc, H. N., Ozgun, U., Sirit, A. (2019). Design, synthesis and application of chiral tetraoxacalix[2] arene[2]triazine-based organocatalysts in asymmetric Michael addition reactions. Chirality, 31, 293-300.

Harutyunyan, S. R., Hartog, T. D., Geurts, K., Minnaard, A. J., Feringa, B. L. (2008). Catalytic asymmetric conjugate addition and allylic alkylation with grignard reagents. Chemical Reviews, 108, 2824-2852.

Hayashi, Y., Gotoh, H., Hayashi, T., Shoji, M. (2005). Diphenylprolinol silyl ethers as efficient organocatalysts for the asymmetric Michael reaction of aldehydes and nitroalkenes. Angewandte Chemie International Edition, 44, 4212-4215.

Li, B-L., Wang, Y-F., Luo, S-P., Zhong, A-G., Li, Z-B., Du, X-H., Xu, D-Q. (2010). Enantioselective michael addition of aromatic ketones to nitroolefins catalyzed by bifunctional thioureas and mechanistic insight. European Journal of Organic Chemistry, $656-662$.

Liu, B., Han, X., Dong, Z., Lv, H., Zhou, H-B., Dong, C. (2013). Highly enantioselective Michael addition of 1,3-dicarbonyl compounds to nitroalkenes catalyzed by designer chiral BINOL-quinine-squaramide: efficient access to optically active nitroalkanes and their isoxazole derivatives. Tetrahedron Asymmetry, 24, 1276-1280.

Lu, A., Hu, K., Wang, Y., Song, H., Zhou, Z., Fang, J., Tang, C. (2012). Enantioselective synthesis of trans-dihydrobenzofurans via primary amine-thiourea organocatalyzed intramolecular Michael addition. The Journal of Organic Chemistry, 77, $6208-6214$.

Ma, H., Liu, K., Zhang, F-G., Zhu, C-L., Nie, J., Ma, J-A. (2010). Chiral bifunctional thiourea-catalyzed enantioselective Michael addition of ketones to nitrodienes. The Journal of Organic Chemistry, 75, 1402-1409.

Mase, N., Thayumanavan, R., Tanaka, F., Barbas III, C. F. (2004). Direct asymmetric organocatalytic Michael reactions of $\alpha, \alpha-$ disubstituted aldehydes with $\beta$-nitrostyrenes for the synthesis of quaternary carbon-containing products. Organic Letters, 6, 25272530.

Mase, N., Watanabe, K., Yoba, H., Takabe, K., Tanaka, F., Barbas III, C. F. (2006). Organocatalytic direct Michael reaction of ketones and aldehydes with $\beta$-nitrostyrene in brine. Journal of the American Chemical Society, 128, 4966-4967.

Naziroglu, H. N., Durmaz, M., Bozkurt, S., Demir, A. S., Sirit, A. (2012). Application of L-prolinamides as highly efficient organocatalysts for the asymmetric Michael addition of unmodified aldehydes to nitroalkenes. Tetrahedron Asymmetry, 23, 164169.

Naziroglu, H. N., Sirit, A. (2016). Novel efficient bifunctional calixarene thiourea organocatalysts: synthesis and application in the direct enantioselective aldol reactions. Tetrahedron Asymmetry, 27, 201-207.

Nugent, T. C., Shoaib, M., Shoaib, A. (2011). Practical access to highly enantioenriched quaternary carbon Michael adducts using simple organocatalysts. Organic \& Biomolecular Chemistry, 9, 52-56.

Palomo, C., Vera, S., Mielgo, A., Go'mez-Bengoa, E. (2006). Highly efficient asymmetric Michael addition of aldehydes to nitroalkenes catalyzed by a simple trans-4-hydroxyprolylamide. Angewandte Chemie International Edition, 45, 5984-5987.

Pansare, S. V., Pandya, K. (2006). Simple diamine and triamine-protonic acid catalysts for the enantioselective Michael addition of cyclic ketones to nitroalkenes. Journal of the American Chemical Society, 128, 9624-9625.

Sulzer-Mossé, S., Alexakis, A. (2007). Chiral amines as organocatalysts for asymmetric conjugate addition to nitroolefins and vinyl sulfonesviaenamine activation. Chemical Communications, 30, 3123-3135.

Tsakos, M., Kokotos, C. G., Kokotos, G. (2012). Primary amine-thioureas with improved catalytic properties for "difficult" Michael reactions: Efficient organocatalytic syntheses of $(S)$-Baclofen, $(R)$-Baclofen and $(S)$-Phenibut. Advanced Synthesis \& Catalysis, 354, 740-746.

Tsandi, E., Kokotos, C. G., Kousidou, S., Ragoussis, V., Kokotos, G. (2009). Sulfonamides of homoproline and dipeptides as organocatalysts for Michael and aldol reactions. Tetrahedron, 65, 1444-1449.

Tsogoeva, S. B. (2007). Recent advances in asymmetric organocatalytic 1,4-conjugate additions. European Journal of Organic Chemistry, 11, 1701-1716.

Wang, M-X., Yang, H-B. (2004). A General and high yielding fragment coupling synthesis of heteroatom-bridged calixarenes and the unprecedented examples of calixarene cavity fine-tuned by bridging heteroatoms. Journal of the American Chemical Society, 126, $15412-15422$. 
Yalalov, D.A., Tsogoeva, S. B., Schmatz, S. (2006). Chiral thiourea-based bifunctional organocatalysts in the asymmetric nitroMichael addition: A joint experimental-theoretical study. Advanced Synthesis \& Catalysis, 348, 826-832.

Yoshida, M., Sato, A., Hara, S. (2010). Asymmetric Michael addition of aldehydes to nitroalkenes using a primary amino acid lithium salt. Organic \& Biomolecular Chemistry, 8, 3031-3036. 